УДК 531.39

\author{
СИЛЫ ВЗАИМОДЕЙСТВИЯ ДОЛОТА С ПОРОДОЙ \\ ПРИ КОМПЬЮТЕРНОМ МОДЕЛИРОВАНИИ ДИНАМИКИ \\ БУРИЛЬНОЙ КОЛОННЫ
}

\title{
BIT-ROCK INTERACTION FORCES IN COMPUTER-AIDED SIMULATION OF DRILLSTRING DYNAMICS
}

\author{
Д.Ю. Погорелов, Н.Н. Лысиков \\ Брянский государственный технический университет, \\ г. Брянск, Российская Федерация \\ Dmitriy Yu. Pogorelov, Nikolay N. Lysikov \\ Bryansk State Technical University, \\ Bryansk, Russian Federation \\ pogorelov@umlab.ru
}

\begin{abstract}
Аннотация. Рассмотрены модели сил взаимодействия долота с породой, которые используются для компьютерного исследования динамики процесса бурения. Приводится подробная модель момента сопротивления бурению, имеющая режимы сцепления и скольжения и позволяющая исследовать автоколебания бурильной колонны. В динамической модели момента могут быть использованы зависимости для стационарных значений момента сопротивления, в частности, значения удельного момента или модель Уоррена для шарошечного долота. Приведена также альтернативная модель момента сопротивления, предложенная Такером и Вангом для исследования крутильных автоколебаний.

В простейшем случае для расчета осевой силы используется упругодиссипативная модель с возможностью добавления гармонических и стохастических возмущений. Поперечные силы также могут быть заданы гар-
\end{abstract}


моническими и случайными функциями времени, параметры которых подбираются на основе экспериментальных данных.

Приведена реализация значительно более сложной модели PDC-долота (модель RGD), учитывающей связь между осевым и угловым движениями долота и позволяющей исследовать процессы колебаний бура с учетом изменения геометрии забоя в процессе моделирования.

В работе предложена уточненная модель шарошечного долота, основанная на расчете сил, возникающих при взаимодействии отдельного зубца с породой.

Приведены примеры использования описанных моделей для исследования автоколебаний и вибраций БК длиной 1 км в криволинейной скважине.

Разработанные модели реализованы в программном комплексе «Универсальный механизм».

Abstract. Models of bit-rock interaction forces in computer-aided simulation of drilling process dynamics are considered in the paper. The torque on bit (TOB) model with sticking and sliding modes is described in details and can be used for analysis of self-excited stick-slip oscillations of drilling process dynamics. Stationary models can be used in the dynamic model of TOB, in particular the specific torque value as well as the Warren's model for a roller-cone bit. An alternative Tucker-Wang TOB model is considered as well for simulation of self-excited oscillations.

A simple force on bit model is presented by a viscous-elastic force model with additional optional harmonic and stochastic oscillations. Lateral forces can be added as harmonic and stochastic time functions which parameters are identified from experimental data.

A considerably more complicated RGF model of drag bit-rock interaction is presented. The RGD model takes into account coupling between axial and torsional bit motions and allows studying the bit bounce with impacts. 
An advanced roller-cone bit model based on single indentor interaction with the rock is proposed in the paper.

A $1 \mathrm{~km}$ drillstring is used for simulation of stick-slip oscillations and vibrations in a curved borehole as examples of application of the described force models.

The discussed models are implemented in the Universal Mechanism software.

Ключевые слова: динамика бурильной колонны, взаимодействие бура с породой, автоколебания, вибрации

Key words: drillstring dynamics, bit-rock interaction, self-excited oscillations, vibrations

\section{Введение}

Развитие методов компьютерного моделирования динамики бурильной колонны (БК) является перспективным и быстро развивающимся направлением вычислительной механики. Динамические модели позволяют оценить нагруженность элементов бурильной колонны, выявить неблагоприятные режимы бурения, выполнить оптимизацию структуры нижней компоновки бурильной колонны и процесса бурения. Адекватность динамической модели определяется двумя основными факторами: корректностью представления упругой колонны с переменным сечением в скважине, центральная линия которой имеет форму пространственной кривой, и обоснованностью моделей взаимодействия бурильной колонны с промывочной жидкостью и породой. В данной статье обсуждаются модели взаимодействия долота с породой, реализованные в программном комплексе «Универсальный механизм» (ПК УМ) [1,2], и приводятся примеры их использования.

Исследованию крутильных автоколебаний БК посвящены многочисленные публикации [3-9]. Колебания возникают вследствие падающей ха- 
рактеристики зависимости момента сопротивления от скорости вращения бурильного инструмента. В данной работе рассмотрены две модели момента: фрикционный момент с режимами сцепления и скольжения [10] и модель Такера-Ванга регуляризированного момента [3].

Расчет вибраций БК, вызванных взаимодействием бурильного инструмента с породой, является важной частью динамического анализа, связанного с оценкой нагруженности элементов нижней компоновки БК. Задача компьютерного моделирования процесса резания и скалывания породы и корректной оценки возникающих при этом сил имеет высокую сложность. Заслуживающей внимания является RGD модель долота режущескалывающего типа [11-13], в частности, PDC-долота. Данная модель является обобщением формул расчета стационарных значений скорости бурения, осевой силы и момента сопротивления на долоте [11] и дает возможность исследовать взаимосвязь осевых и крутильных колебаний долота с учетом истории процесса. В частности, RGD модель может быть использована для исследования процессов развитие осевых колебаний долота с возможным отрывом от породы. В данной работе приводятся краткое описание RGD модели и пример ее использования для расчета динамики БК.

В последнем разделе статьи приведена оригинальная многомассовая модель шарошечного долота. Взаимодействие долота с породой моделируется на основе экспериментальных характеристик для сил сопротивления внедрению отдельного зубца в породу. Модель позволяет рассчитывать все компоненты сил, действующих на долото со стороны породы: осевую силу, момент сопротивления и поперечные воздействия, а также качественно и количественно оценивать влияние на силы геометрических параметров долота и свойств породы. 


\section{Тестовая модель бурильной колонны}

Для выполнения тестовых расчетов в рамках данной статьи автоматически сгенерирована модель упругой бурильной колонны с использованием ПК УМ. Колонна имеет общую длину 1000 м, нижняя компоновка 170 м (рисунок 1).

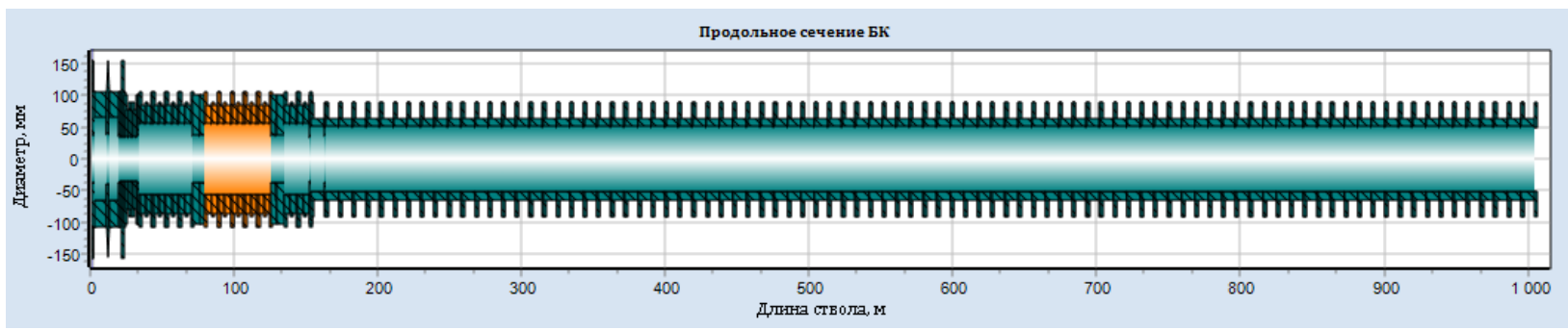

Рисунок 1. Схема бурильной колонны

БК размещается в криволинейной скважине переменного сечения: осевая линия имеет вертикальную секцию длиной 500 м и криволинейную часть постоянной кривизной протяженностью 200 м с выходом на горизонтальный участок (рисунок 2).

Динамическая модель БК содержит 423 геометрически нелинейных балочных конечных элементов $[14,15]$ и имеет 2544 степени свободы. Моделирование динамики происходит в положении, указанном на рисунке 2. Из положения равновесия, изображенного на рисунке 2 , колонна приводится во вращение моментом $M_{c}$, приложенным к верхней части.

Реализуется пропорционально-интегральное управление по угловой скорости

$$
M_{c}=K_{\mathrm{P}}\left(\omega_{c}(t)-\omega\right)+K_{\mathrm{I}} \int_{0}^{t}\left(\omega_{c}(t)-\omega\right) d t
$$

с постоянными коэффициентами $K_{\mathrm{P}}, K_{\mathrm{I}} ; \omega, \omega_{c}(t)$ - текущее и задаваемое значение угловой скорости верхней части БК. 

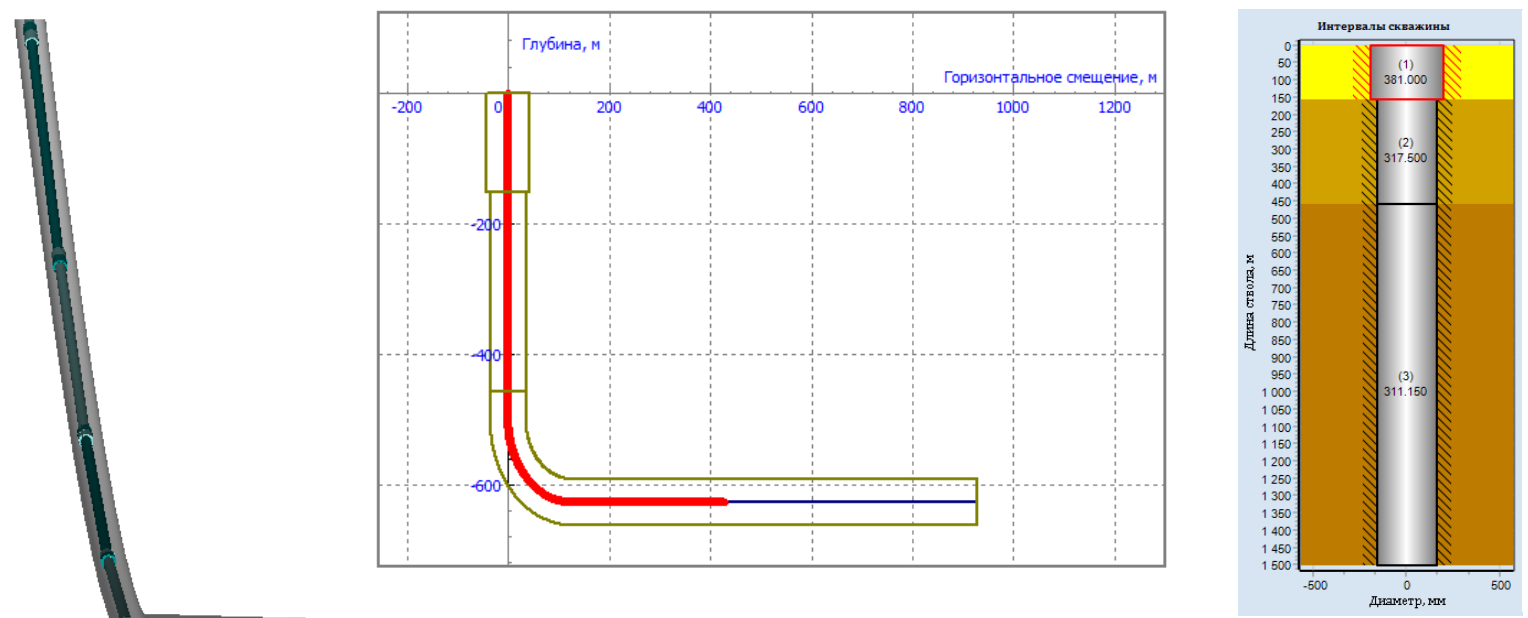

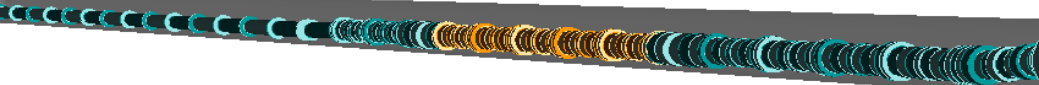

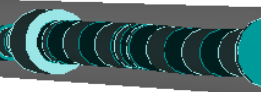

Рисунок 2. Модель бурильной колонны в криволинейной скважине

В примерах, приведенных ниже, принята следующая задавающая угловая скорость:

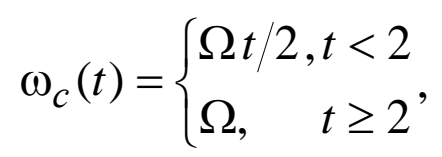

то есть угловая скорость в течение двух секунд равноускоренно увеличивается до значения $\Omega$.

\section{Гармонические и стохастические возмущающие воздействия}

Модели взаимодействия бура с породой, описанные ниже в данной статье, являются в известной мере упрощенными. Например, модели момента и осевой силы не определяют боковые воздействия на долото. В соответствии с работами [16, 17], анализ экспериментальных данных по боковым воздействиям на бур показал, что для шарошечных долот поперечные воздействия имеют случайный характер, практически - белый шум, а для PDC долот - ярко выраженный гармонический закон изменения. Поэтому полезной является возможность добавления стохастических 


$$
F(t)=F_{0} \xi(t)
$$

и гармонических воздействий

$$
F(t)=F_{0} \sin (\omega t+\delta)
$$

явным образом зависящих от времени.

Случайная функция $\xi(t)$ генерируется по спектральной плотности мощности в заданном диапазоне частот. На рисунке 3 представлен график белого шума, спектральная плотность которого имеет единичное значение в диапазоне частот $[0,50]$ рад/с.

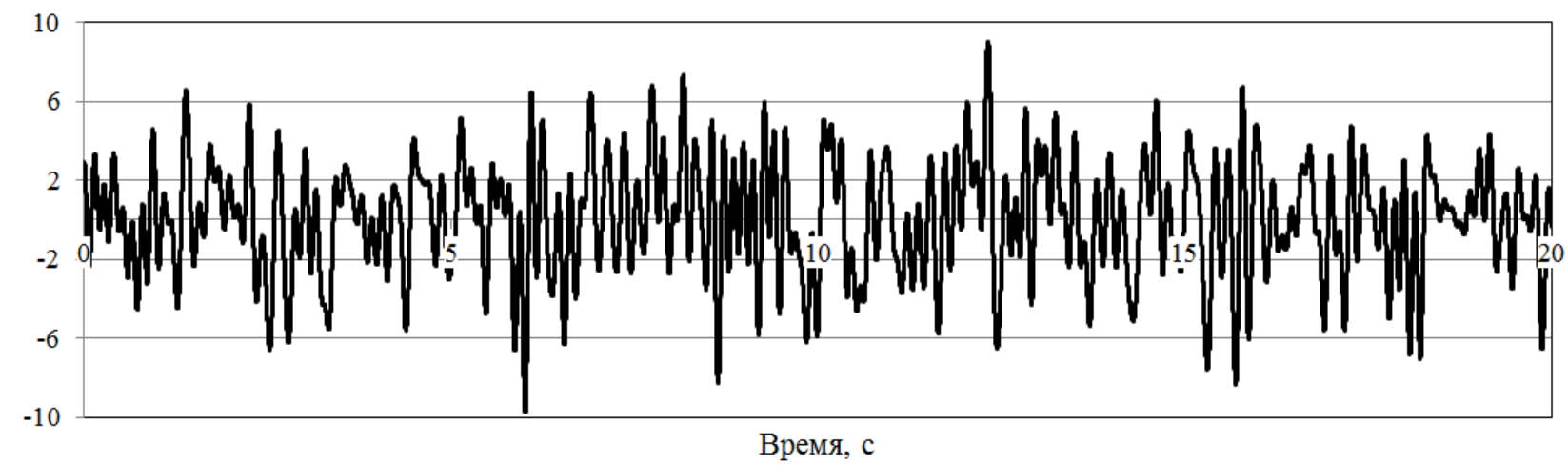

Рисунок 3. Фрагмент случайной функции времени - белого шума

Модель осевой силы может включать гармоническую и стохастическую составляющие, вызванные отклонением от идеальной формы поверхности забоя. Для глубины резания в этом случае принимается следующая зависимость:

$$
\Delta=\left(z_{b 0}-z_{b}\right)+a_{h} \sin \left(n \varphi_{b}\right)+a_{s} \xi(t),
$$

где $z_{b}$ - текущая осевая координата долота;

$z_{b 0}$ - осевая координата идеальной поверхности забоя;

$a_{h}, a_{s}-$ амплитуды гармонической и стохастической составляющей отклонения поверхности от идеальной;

$n$ - число гармоник возмущения на один оборот долота (например число шарошек). 


\section{Упрощенная модель осевой силы}

В упрощенном случае в осевом направлении нагрузка на долото определяется односторонней упруго-диссипативной контактной силой

$$
G=\left\{\begin{array}{l}
G_{c}=c_{w} \Delta+v_{w} d \Delta / d t, \Delta>0, G_{c}>0 \\
0, \Delta<0, G_{c}<0
\end{array},\right.
$$

где $c_{w}, v_{w}$ - коэффициенты жесткости и демпфирования упруго-вязкого основания;

$\Delta$ - глубина резания (1).

Поскольку в данном случае осевое смещение породы в результате разрушения не предусматривается, то глубина резания, фактически, является упругим прогибом опорной поверхности. Более сложные модели осевой силы рассмотрены ниже при описании моделей RGD и шарошечного долота с многоточечным взаимодействием с породой.

\section{Модель фрикционного момента на долоте}

Момент сопротивления вращению долота моделируется фрикционным моментом $M_{f}$ и реализует два режима: скольжение и сцепление [10]. В режиме скольжения момент определяется формулой

$$
M_{f}=-\mu\left(\omega_{b}\right) G R_{b} \frac{\omega_{b}}{\left|\omega_{b}\right|}=-m G \frac{\omega_{b}}{\left|\omega_{b}\right|} .
$$

Здесь $R_{b}$ - радиус долота, $\mu\left(\omega_{b}\right)$ - коэффициент сопротивления вращению, зависящий от угловой скорости долота $\omega_{b}, m=\mu R_{b}-$ удельный момент сопротивления [18-20], G - осевая нагрузка на долото.

Для расчета коэффициента сопротивления вращению используется формула, применяющаяся при описании трения скольжения, являющаяся комбинацией экспоненциальной модели, учитывающей кривую Штрибека [21], и вязкого трения: 


$$
\mu(\omega)=\mu_{\infty}+\left(\mu_{0}-\mu_{\infty}\right) e^{-\left(\frac{\omega}{\omega^{*}}\right)^{\delta}}+v \omega .
$$

В данной зависимости $\mu_{0}$ - значение коэффициента сопротивления при нулевой угловой скорости (аналог коэффициент трения сцепления), $\mu_{\infty}$ - коэффициент сопротивления при бесконечной угловой скорости, $\omega^{*}$ - скорость Штрибека, определяющая величину интервала скорости проскальзывания, на которой имеет место эффект Штрибека экспоненциального падения коэффициента, $\delta \in[0.5,1]$ - эмпирический показатель степени, $v$ - коэффициент вязкого трения.

Приведенная зависимость позволяет реализовать множество моделей, используемых на практике (рисунок 4):

a) $\mu_{\infty}=\mu_{0}, \omega^{*}=0, v=0$ - классическая модель кулоновского трения с коэффициентом трения скольжения равным коэффициенту трения покоя;

б) $\mu_{\infty}<\mu_{0}, \omega^{*}=0, v=0$ - классическая модель сухого трения с коэффициентом трения скольжения меньше коэффициента трения покоя;

в) $\mu_{\infty}=\mu_{0}, \omega^{*}=0, v>0$ - дополнительно к случаю а) учитывается вязкое трение;

г) $\mu_{\infty}<\mu_{0}, \omega^{*}=0, v>0$ - дополнительно к случаю б) учитывается вязкое трение;

д) $\mu_{\infty}<\mu_{0}, \omega^{*}>0, v=0$ - модель Штрибека;

е) $\mu_{\infty}<\mu_{0}, \omega^{*}>0, v>0$ - модель Штрибека с учетом вязкого трения.

Переход от режима скольжения к режиму сцепления осуществляется, когда угловая скорость меняет свое направление на противоположное. В этот момент запоминаются значения момента скольжения (обозначим его $M_{f s}$ ) и угла поворота породоразрушающего инструмента $\varphi_{b s}$. 


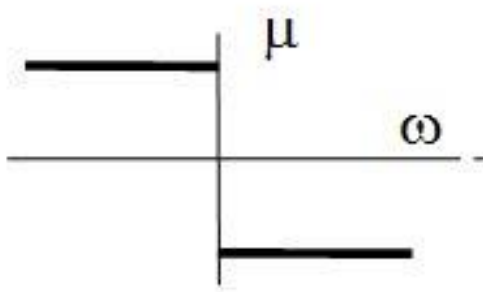

a)

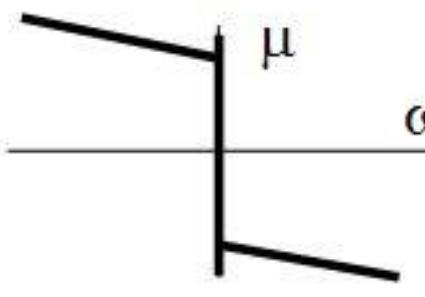

г)

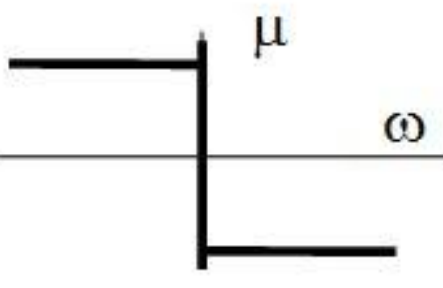

6)

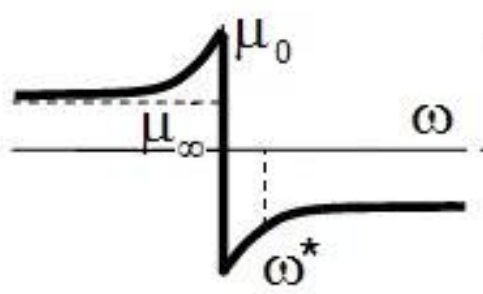

д)

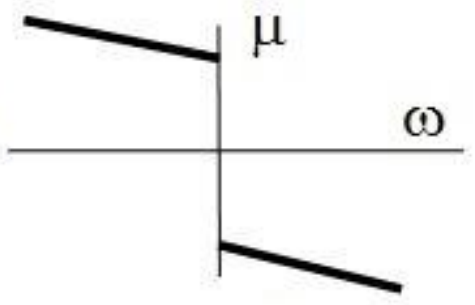

в)

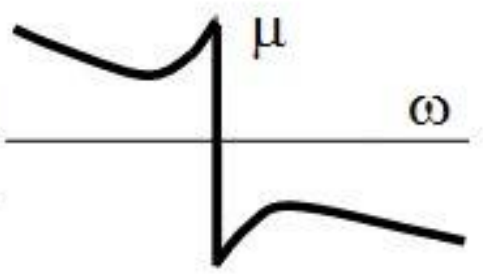

e)

Рисунок 4. Модели зависимостей коэффициента трения от скорости проскальзывания

В режиме сцепления используется следующая модель:

$$
M_{f}=M_{f s}-c\left(\varphi_{b}-\varphi_{b s}\right)-d \omega_{b},
$$

где $\varphi_{b}$ - текущее значение угла поворота долота.

Таким образом, при переходе к сцеплению долото «привязывается»к породе линейным упруго-диссипативным элементом. Слагаемое $M_{f s}$ в формуле обеспечивает непрерывность момента при переходе от скольжения к сцеплению.

Режим сцепления заканчивается при достижении момента сопротивления предельного значения, то есть при выполнении условия

$$
\left|M_{f}\right|>\mu_{0} G R_{b}
$$

При потере контакта инструмента с породой, то есть при $G=0$, момент трения полагается равным нулю.

Пример использования данной модели момента для исследования крутильных автоколебаний бурильной колонны приведен на рисунке 5.

Расчеты выполнены при нагрузке на долото $G=100 \kappa Н$. График момента соответствует угловой скорости $\Omega=20$ об/мин. 

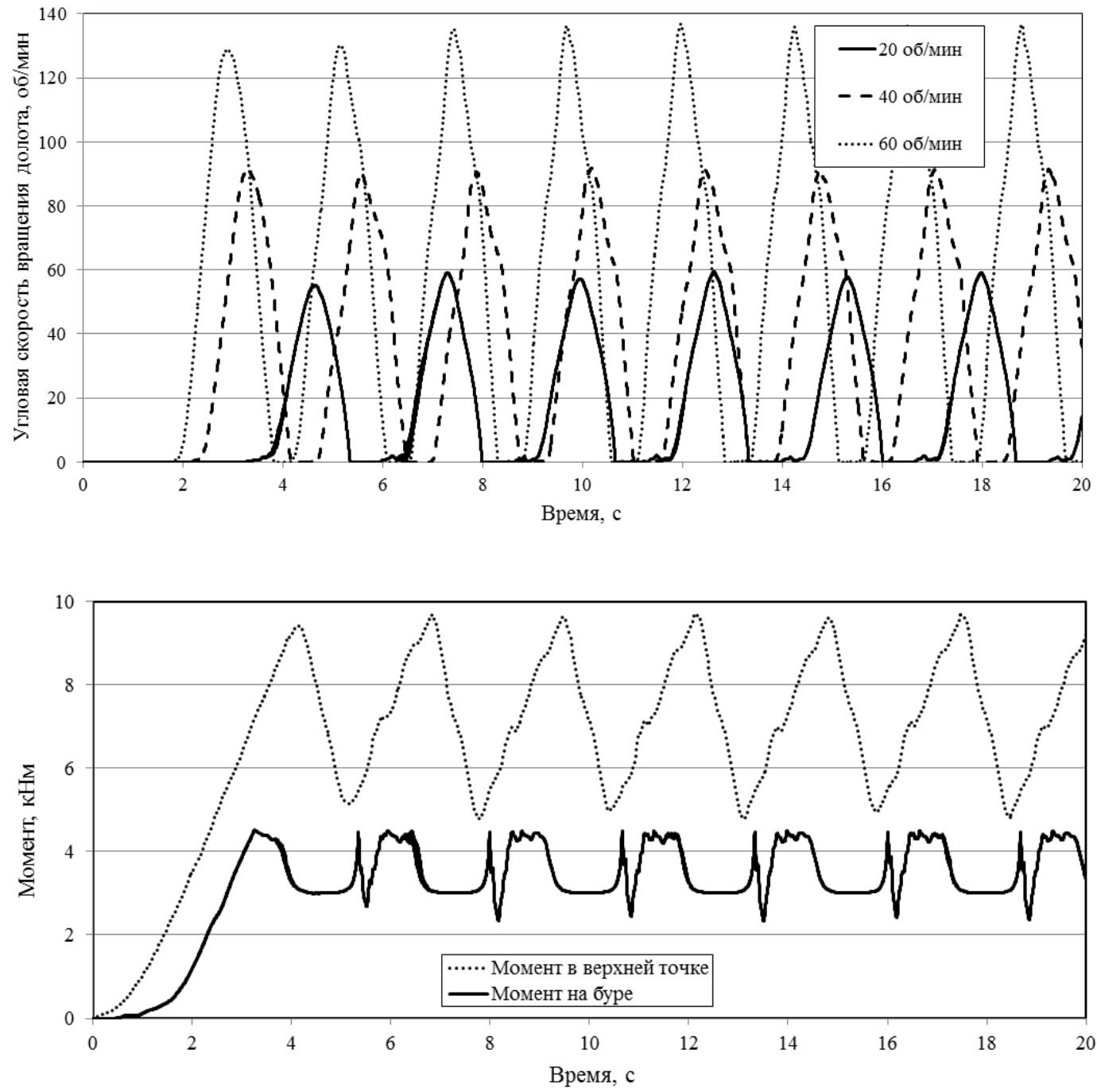

Рисунок 5. Расчет крутильных автоколебаний с использованием модели фрикционного момента

\section{Стационарные модели момента на долоте}

Стационарные или установившиеся модели момента сопротивления получили широкое распространение в теории бурения скважин. Эти модели соответствуют установившейся скорости вращения бура и позволяют оценить значение коэффициента сопротивления вращению $\mu_{\infty}$ и соответствующее ему значение удельного момента $m_{\infty}=\mu_{\infty} R_{b}$ в формуле (2).

Сначала рассмотрим модель удельного момента, принятую в советской школе теории бурения. В уточненной постановке удельный момент зависит от осевой нагрузки [18] 


$$
m_{\infty}=A_{1}+A_{2} G
$$

однако при практическом применении часто опускают второе слагаемое, момент считается постоянным, как, например, в модели Г.И. Булаха. Численные значения удельного момента или коэффициента сопротивления для различных типов долот и породы приведены в работах [18-20]. Например, в пособии [19] для шарошечных высокооборотных долот указан интервал $\mu_{\infty} \in[0.042,0.095]$.

Следующая модель для шарошечного долота включает эмпирические нелинейные зависимости для стационарных значений момента сопротивления на долоте и глубины резания $d$ за оборот, предложенные в работах Уоррена [22, 23]

$$
\begin{aligned}
& d^{-1}=\left(\frac{a S^{2} D_{b}^{3}}{G^{2}}+\frac{c}{D_{b}}\right) \\
& m_{\infty}=\left(C_{1}+C_{2} \sqrt{d / D_{b}}\right) D_{b} .
\end{aligned}
$$

В этих формулах $D_{b}=2 R_{b}$ - диаметр долота, $a, b, C_{1}, C_{2}$ - эмпирические постоянные, $\mathrm{S}$ - твердость породы.

\section{Модель Такера-Ванга момента на долоте}

В работе [3] Такер и Ванг предложили вариант модели зависимости установившихся значений момента на долоте $M_{f 0}$ и скорости бурения $v_{0}$ от угловой скорости инструмента и осевой нагрузки

$$
\begin{aligned}
& v_{0}=-a_{1}+a_{2} G+a_{3} \Omega, \\
& M_{f 0}=a_{4} v_{0} / \Omega+a_{5} .
\end{aligned}
$$

Здесь $a_{1}, \ldots, a_{5}$ - эмпирические постоянные;

$\Omega$ - угловая скорость инструмента в оборотах в минуту. 
Для численного моделирования динамических процессов авторы выполнили регуляризацию выражений для скорости бурения и момента треНия

$$
\begin{aligned}
& v=F^{2}\left(-a_{1}+a_{2} G\right)+a_{3} \Omega F, \\
& M_{f}=a_{4} F^{2} v / \Omega+a_{5} F,
\end{aligned}
$$

где введена регуляризирующая функция скорости вращения

$$
F(\Omega)=\frac{\Omega}{\sqrt{\Omega^{2}+\varepsilon^{2}}} .
$$

На рисунке 6 приведены графики момента для различных осевых нагрузок при значениях параметров, указанных в статье [3]

$a_{1}=3.429 \cdot 10^{-3} \mathrm{M} / \mathrm{c}, a_{2}=5.672 \cdot 10^{-8} \mathrm{M} / \mathrm{H} / \mathrm{c}, a_{3}=1.374 \cdot 10^{-4} \mathrm{M}, a_{4}=9.537 \cdot 10^{6} \mathrm{H}$, $a_{5}=1.475 \cdot 10^{3} \mathrm{HM}, \varepsilon=3$.

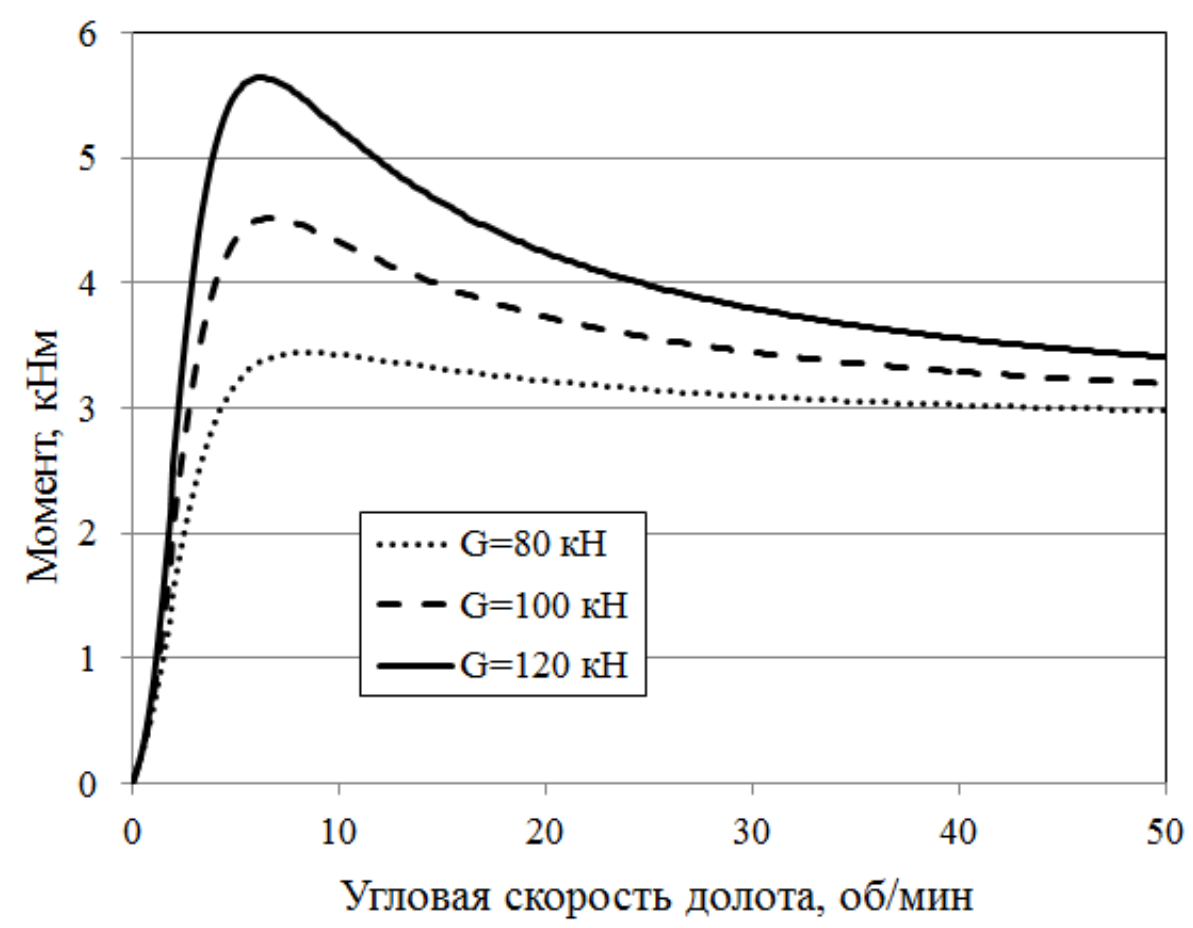

Рисунок 6. Зависимость момента на долоте от угловой скорости по модели Такера-Ванга 
Пример применения данной модели момента для исследования крутильных автоколебаний БК приведен на рисунке 7. Расчет выполнен при $G=100 \kappa Н, \Omega=20$ об/мин.

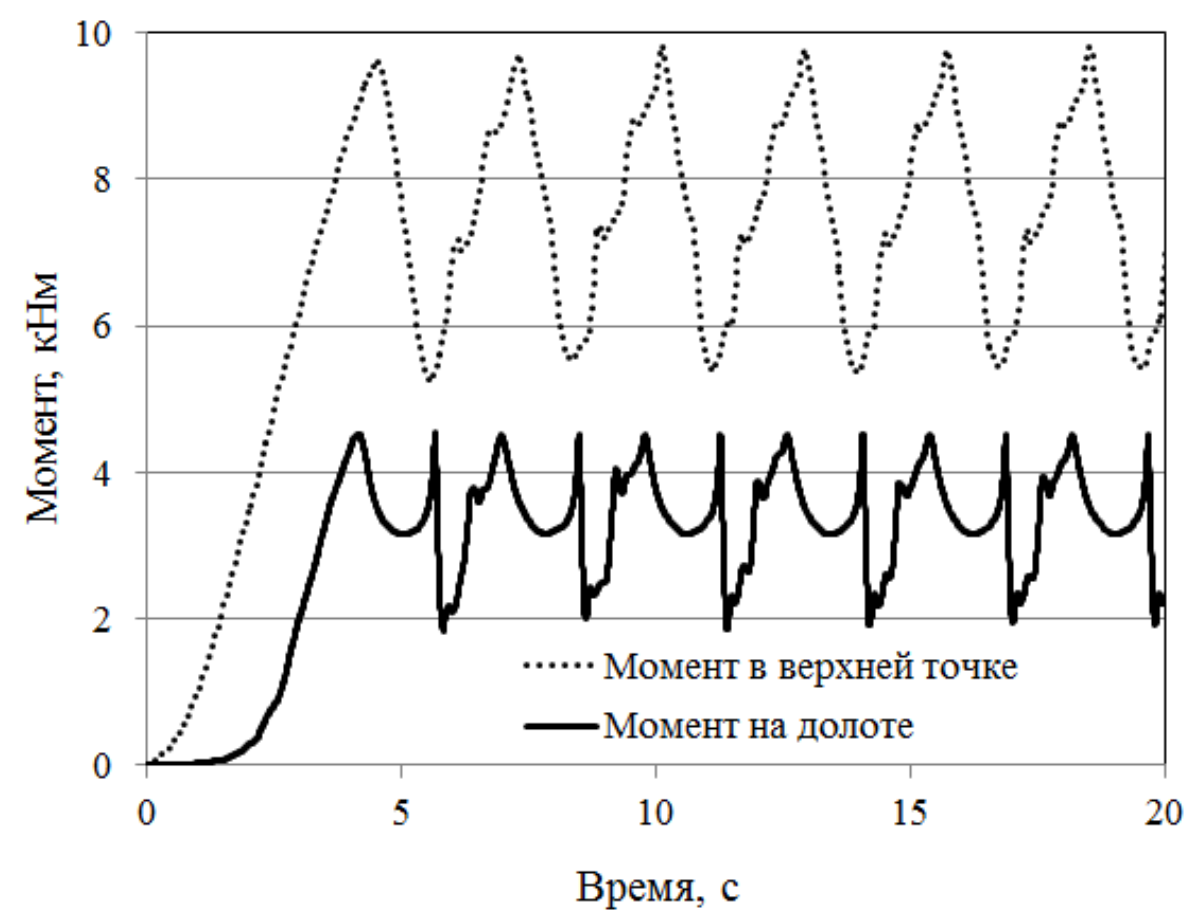

Рисунок 7. Зависимость от времени момента на долоте по модели Такера-Ванга и управляющего момента при автоколебаниях бурильной колонны

\section{RGD модель долота режуще-скалывающего типа}

Модель RGD (T. Richard, C. Germay, E. Detournay) долота режущескалывающего типа описана в работах $[12,13]$. Особенностью этой модели является связь между осевым и угловым движениями долота. Приведем основные соотношения. Осевая сила и момент являются суммой режущей составляющей и трения

$$
G=G_{c}+G_{f}, M=M_{c}+M_{f} .
$$

Составляющие резания вычисляются по формулам [11]

$$
G_{c}=R_{b} \zeta \varepsilon d(t), M_{c}=R_{b}^{2} \varepsilon d(t) / 2,
$$


где $\varepsilon-$ характерная величина энергии, необходимая для резания, зависящая от породы;

$\zeta$ - постоянная, определяемая наклоном силы резания к лезвию;

$d=n d_{n}-$ суммарная глубина резания $n$ лезвиями;

$R_{b}-$ радиус долота.

В установившемся режиме величина $d$ равна глубине резания за один полный оборот долота.

В общем случае эта величина зависит от времени и определяется «историей» динамического процесса взаимодействия инструмента с породой.

Пусть $z_{b}$ - осевая координата, определяющая положение долота в скважине, например расстояние от поверхности до долота вдоль оси скважины. Предполагается, что форма поверхности скважины в осевом направлении полностью определяется поворотом долота, таким образом, что текущая глубина резания отдельным лезвием вычисляется по формуле

$$
d_{n}=d(t) / n=z_{b}\left(\varphi_{b}(t)\right)-z_{b}\left(\varphi_{b}(t)-2 \pi / n\right),
$$

т.е. равна разнице между осевой координатой инструмента в данный момент времени и запомненнылм значением координаты в предыдущем положении, когда лезвия занимали такое же угловое положение (поворот против вращения на угол $2 \pi / n)$. Предполагается, что глубина резания для всех лезвий одинаковая, т.е. равна разнице между осевой координатой инструмента в данный момент времени и положением на предыдущем обороте инструмента (поворот против вращения на угол $2 \pi / n)$. Предполагается, что глубина резания для всех $n$ лезвий одинаковая.

Для расчета силы и момента трения в режиме проскальзывания используются зависимости [11]

$$
G_{f}=R_{b} l \sigma, 2 M_{f}=R_{b} \gamma \mu G_{f}
$$

Здесь $l=n l_{n}-$ суммарная толщина лезвий; 
$\gamma \in[1,4 / 3]-$ параметр, характеризующий геометрию лезвия;

$\sigma-$ контактное давление, примерно равное $\varepsilon$ [12].

Данная модель силы и момента позволяет исследовать не только процесс крутильных автоколебаний БК, но и осевых колебаний, в том числе динамический отрыв бурильного инструмента от породы. Пример моделирования процесса развития осевых колебаний представлен на рисунке 8. Средняя нагрузка на долоте $G=100$ кН, угловая скорость $\Omega=80$ об/мин.
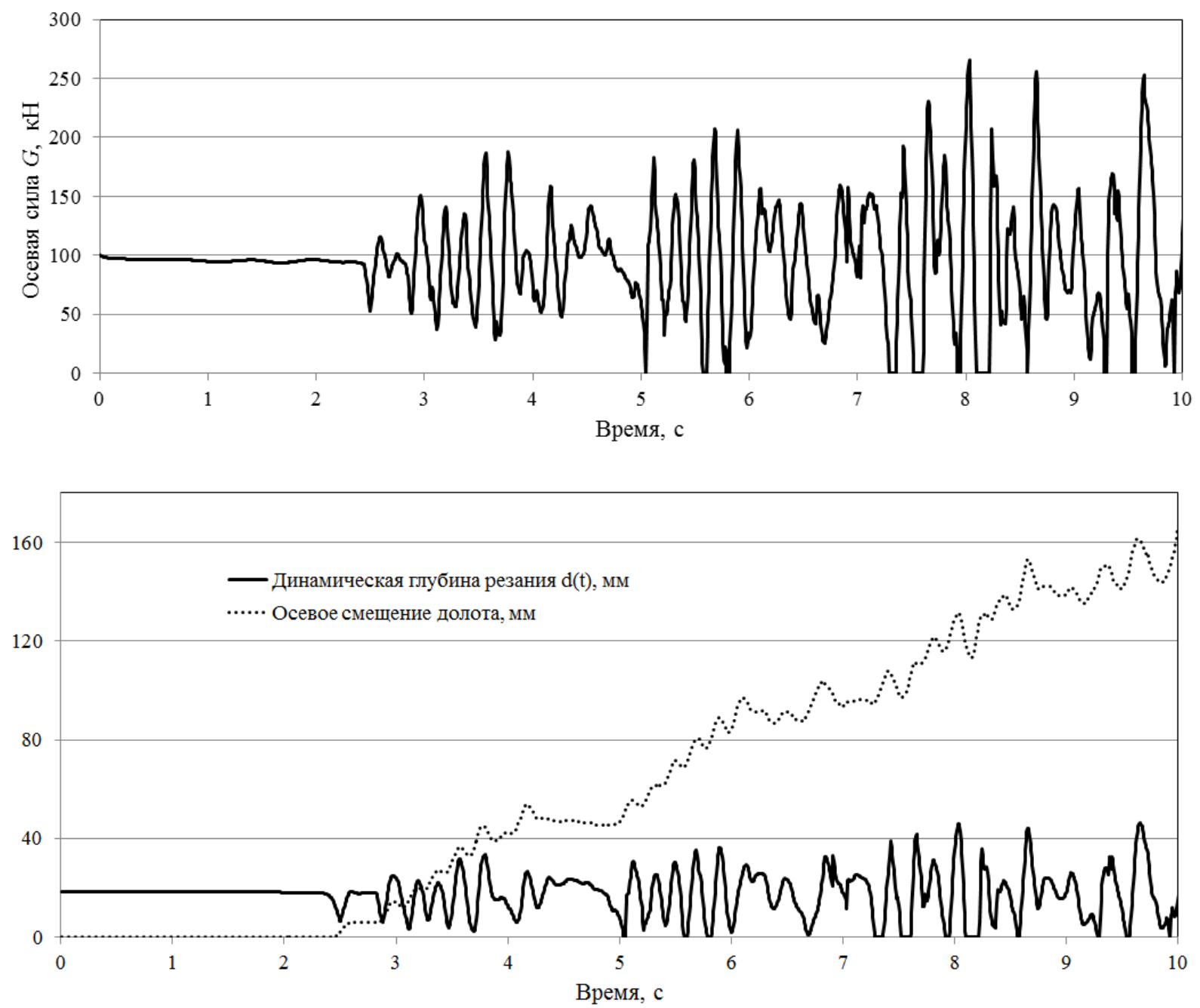

Рисунок 8. Развитие осевых колебаний с отрывом долота при использовании RGD модели 


\section{Уточненная модель шарошечного долота}

В программном комплексе «Универсальный механизм» разработана и реализована оригинальная модель шарошечного долота. Модель позволяет автоматически назначать число шарошек, венцов и зубцов в каждой шарошке, а также геометрические размеры. Пример модели трехшарошечного долота приведен на рисунке 9.

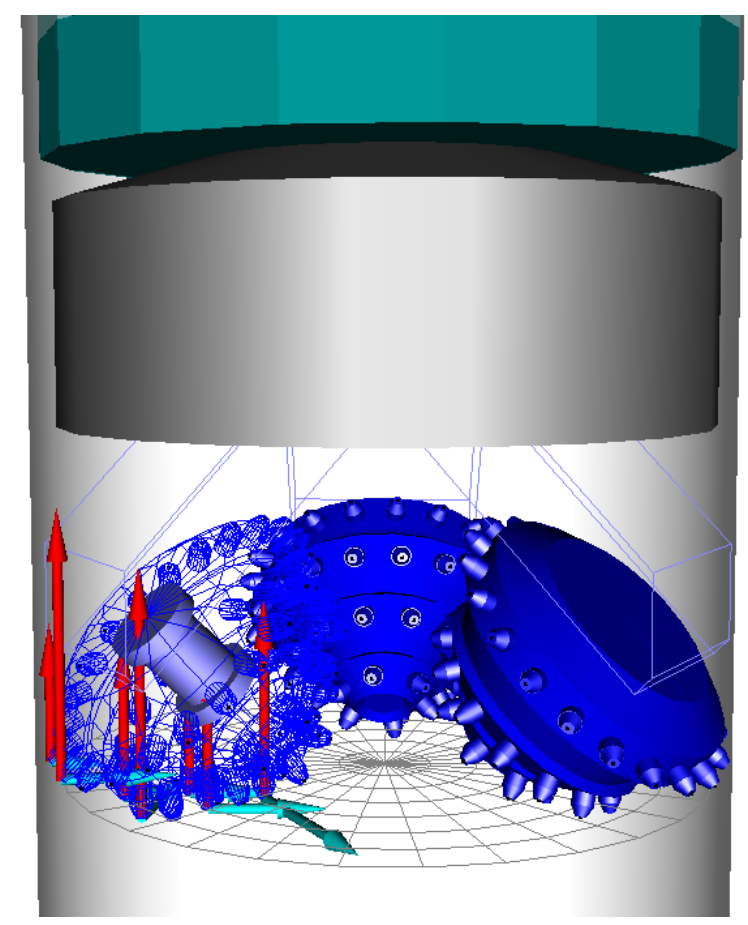

Рисунок 9. Модель трехшарошечного долота. Нормальные и касательные силы взаимодействия зубцов с породой

Особенностью модели является расчет сил взаимодействия с породой для каждого зубца в отдельности. Шарошки имеют по одной вращательной степени свободы относительно корпуса, так что при бурении шарошка катится, и каждый зубец периодически входит в контакт с породой. Модель силового взаимодействия отдельного зубца $i$ с породой основана на результатах статьи [24]. Сила взаимодействия имеет две составляющие: нормальную $G_{i n}$ и касательную $G_{i t}$ (рисунок 10 ). 


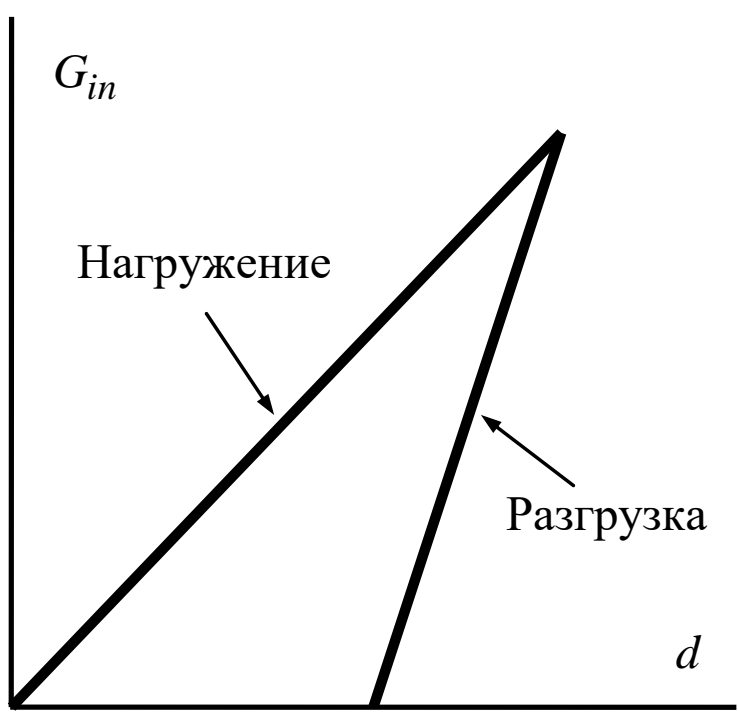

a)

$$
G_{i t}
$$

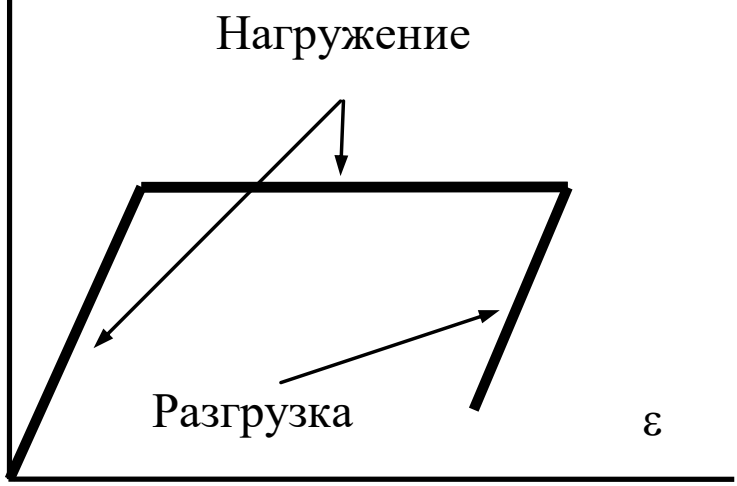

б)

Рисунок 10. Модели нормальной и касательной сил, действующих на отдельный зубец шарошки

При нагружении, т.е. при увеличении глубины $d$ проникновения зубца в породу, нормальная сила линейно растет с коэффициентом пропорциональности $\sigma$

$$
G_{i n}=\sigma d .
$$

При разгрузке (уменьшение $d$ ) нормальная сила убывает также по линейному закону с коэффициентом пропорциональности $\alpha \sigma$

$$
G_{i n}=\alpha \sigma d .
$$

В работе [24] на основании эксперимента предложено значение $\alpha=5$.

Для касательной силы в статье [24] рассмотрена упруго-пластическая модель зависимости от поперечного смещения зубца $\varepsilon$. На этапе нагружения модель силы следующая (рисунок 10, б):

$$
G_{i t}=\left\{\begin{array}{ll}
\mu d \varepsilon / \varepsilon_{0}, & \varepsilon<\varepsilon_{0} \\
\mu d, & \varepsilon \geq \varepsilon_{0}
\end{array} .\right.
$$

Приводится экспериментальная оценка величины упругого смещения $\varepsilon_{0} \sim 0.1 \mathrm{MM}$. 
При разгрузке используется линейная модель касательной силы с такой же жесткостью, как и при нагружении.

Данная модель шарошечного долота позволяет качественно и количественно исследовать зависимость сил, действующих на долото как в осевом, так и поперечном направлении, при изменении геометрических параметров долота и свойств породы.

Типичные зависимости от времени осевой силы и момента на долоте, полученные в соответствии с описанной моделью трехшарошечного долота в результате компьютерного моделирования динамики процесса бурения показаны на рисунке 11. Средняя нагрузка на долоте $G=100$ кН, угловая скорость $\Omega=60$ об/мин.
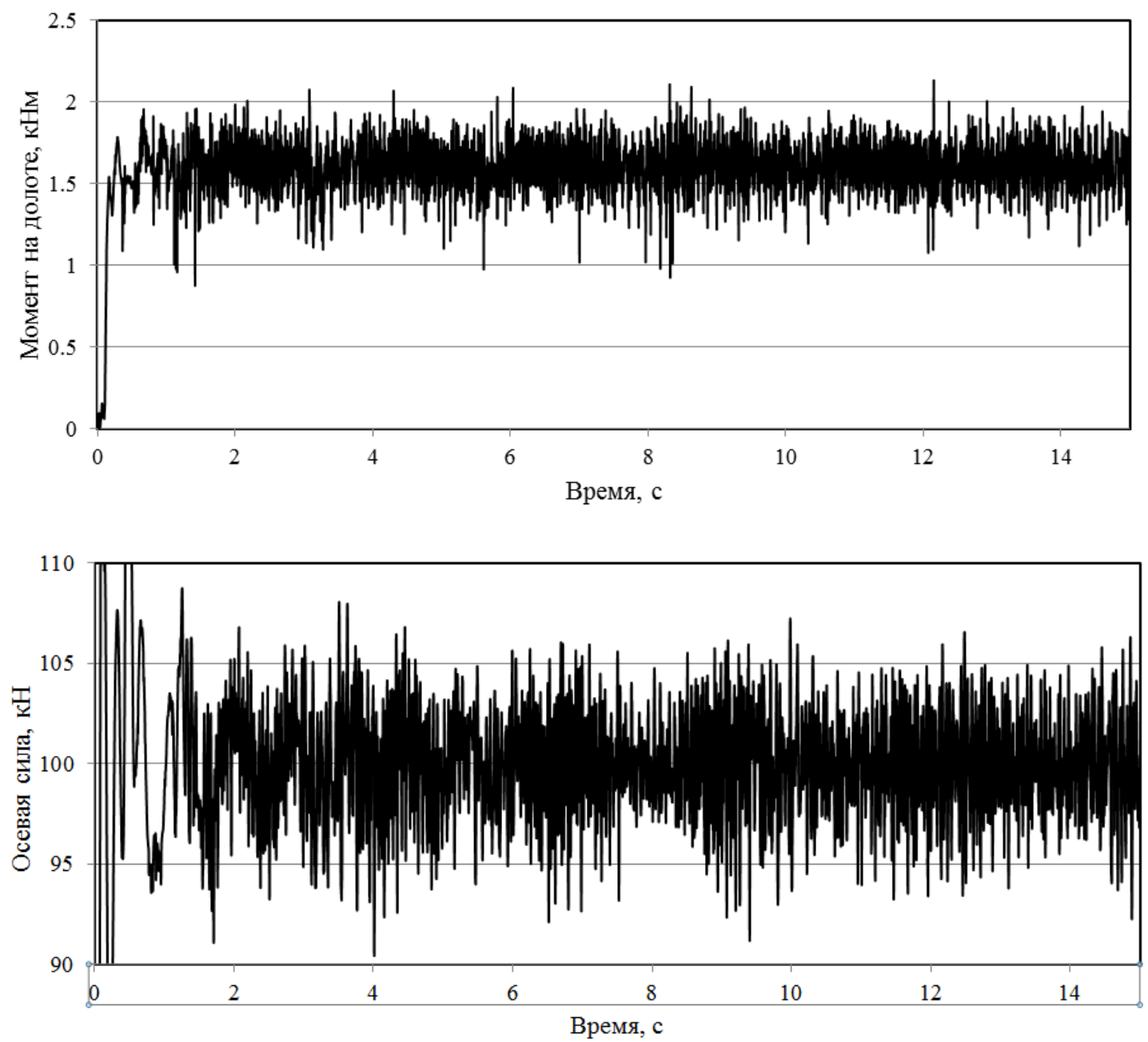

Рисунок 11. Пример взаимодействия уточненной модели трехшарошечного долота и породы 


\section{Выводы}

1. Приведенные моделей взаимодействия долот различных типов с породой позволяют исследовать динамические процессы при бурении. Все модели момента сопротивления показывают возможность возникновение крутильных автоколебаний БК. RGD модель учитывает взаимосвязь крутильных и осевых колебаний долот скалывающего-режущего типа; численный пример демонстрирует возникновение осевых колебаний с отрывом бура. Модель шарошечного долота с расчетом взаимодействий отдельных зубцов с породой позволяет рассчитывать все компоненты сил и может применяться для оценки вибронагруженности элементов нижней компоновки БК.

2. Для обоснованного выбора параметров моделей в зависимости от типа долота и свойств породы требуются дополнительные исследования, связанные с анализом экспериментальных данных.

Работа выполнена при поддержке РФФИ (грант № 17-01-00815a).

\section{Список используемых источников}

1. Погорелов Д.Ю. Компьютерное моделирование динамики технических систем с использованием программного комплекса «Универсальный механизм» // Вестник компьютерных и информационных технологий. 2005. № 4. С. 27-34.

2. Сайт «Универсальный механизм». URL: http://www.universalmechanism.com (дата обращения: 07.02.2019).

3. Tucker R.W., Wang. C. Torsional Vibration Control and Cosserat Dynamics of a Drill-Rig Assembly // Meccanica. 2003. Vol. 38. No.1. pp. 145161. DOI: https://doi.org/10.1023/A:102203582. 
4. Christoforou A.P., Yigit A.S. Fully Coupled Vibrations of Actively Controlled Drill Strings // Journal of Sound and Vibrations. 2003. Vol. 267. No. 5. pp. 1029-1045. DOI: 10.1016/S0022-460X(03)00359-6.

5. Sampaio R., Piovan M. T., Venero Lozano G. Coupled Axial/Torsional Vibrations of Drill Strings by Means of Non-Linear Model // Mechanics Research Communications. 2007. Vol. 34. No. 5-6. pp. 497-502. DOI: 10.1016/j.mechrescom.2007.03.005.

6. Khulief Y.A., Al-Sulaiman F.A., Bashmal S. Vibration Analysis of Drill Strings with Self-Excited Stick-Slip Oscillations // Journal of Sound and Vibration. 2007. Vol. 299. No. 3. pp. 540-558. DOI: 10.1016/j.jsv.2006.06.065.

7. Юнин Е.К. Низкочастотные колебания бурильного инструмента. М.: Недра, 1983. 132 с.

8. Пальмов В.А., Ветюков Ю.М. Неравномерное вращение бурильной колонны, обусловленное существенно нелинейным взаимодействием долота со скальной породой // Научно-технические ведомости СПбГТУ. 2003. № 4. C. 51-56.

9. Коронатов В.А. Новая динамическая модель бурильной колонны и численное описание крутильных автоколебаний во время проходки углубления забоя скважины // Системы. Методы. Технологии. 2015.№ 1 (25). C. 48-59.

10. Погорелов Д.Ю., Павлюков А.Э., Юдакова Т.А., Котов С.В. Моделирование контактных взаимодействий в задачах динамики систем тел // Динамика, прочность и надежность транспортных машин: сб. науч. тр. Брянск: БГТУ, 2002. С. 11-23.

11. Detournay E., Defourny P. A Phenomenological Model for the Drilling Action of Drag Bits // Int. J. Rock Mech. Min. 1992. Vol. 29. No. 1. pp. 13-23.

12. Richard T., Germay C., Detournay E. Self-Excited Stick-Slip Oscillations of Drill Bits // Mecanique. 2004. Vol. 332. pp. 619-626. DOI: https://doi.org/10.1016/j.crme.2004.01.016. 
13. Richard T., Germay C., Detournay E. A Simplified Model to Explore the Root Cause Of Stick-Slip Vibrations in Drilling Systems with Drag Bits // Journal of Sound and Vibration. 2007. Vol. 305. No. 3. pp. 432-456. DOI: https://doi.org/10.1016/j.jsv.2007.04.015.

14. Pogorelov D., Mikheev G., Lysikov N., Ring L., Gandikota R., Abedrabbo N. A Multibody System Approach to Drill String Dynamics Modeling // Proc. ASME 11th Biennial Conference on Engineering Systems Design and Analysis (ESDA2012). France, 2012. Vol. 4. pp. 53-62. DOI: 10.1115/ESDA2012-82316.

15. Dmitrochenko O., Mikheev G., Pogorelov D., Gandikota R. A Nonlinear Finite Element for Simulation of Dynamics of Beam Structures Using Multibody System Approach // Proc. 11th World Congress on Computational Mechanics (WCCM XI). Spain, 2014. pp. 655-666.

16. Brakel J.D. Prediction of Wellbore Trajectory Considering Bottom Hole Assembly and Drillbit Dynamics // PhD Thesis. University of Tulsa, 1986.

17. Spanos P.D., Chevallier A.M., Politis N.P. Nonlinear Stochastic DrillString Vibrations // Journal of Vibration and Acoustics. 2002. Vol. 124. No. 4. pp. 512-518. DOI: https://doi.org/10.1115/1.1502669.

18. Григорян Н.А., Багиров Р.Е. Анализ процесса турбинного бурения. М.: Недра, 1982. 207 с.

19. Спивак А.И., Попов А.Н. Разрушение горных пород при бурении скважин. М.: Недра, 1994. 264 с.

20. Калинин А.Г., Левицкий А.3., Мессер А.Г., Соловьев Н.В. Практическое руководство по технологии бурения скважин на жидкие и газообразные полезные ископаемые. М.: Недра, 2001. 450 с.

21. Stribeck R. Die wesentlichen eigenschaften der Gleit- und Rollenlager // VDI-Zeitschrift. 1902. Vol. 46. No. 36. pp. 1341-1348; 1432-1438; 1463-1470.

22. Warren T.M. Penetration-Rate Performance Of Roller-Cone Bits // SPE Drilling Engineering. 1987. Vol. 2. No. 1. pp. 9-18. DOI: https://doi.org/10.2118/13259-PA. 
23. Warren T.M. Factors Affecting Torque for a Roller Cone Bit // Journal of Petroleum Technology. 1984. Vol. 36. No. 9. pp. 1500-1508. DOI: https://doi.org/10.2118/11994-PA.

24. Sheppard M.C., Lesage M. The Forces at the Teeth of a Drilling Roller Cone Bit: Theory and Experiment // Society of Petroleum Engineers. 1988. SPE-18042. pp. 253-260.

\section{References}

1. Pogorelov D.Yu. Komp'yuternoe modelirovanie dinamiki tekhnicheskikh sistem $\mathrm{s}$ ispol'zovaniem programmnogo kompleksa «Universal'nyi mekhanizm» [Computer Simulation of Technical System Dynamics with Universal Mechanism Software]. Vestnik komp'yuternykh $i$ informatsionnykh tekhnologii - Herald of Computer and Information Technologies, 2005, No. 4, pp. 27-34. [in Russian].

2. Sait «Universal'nyi mekhanizm» [Universal Mechanism Website]. Available at: http://www.universalmechanism.com (accessed 07.01.2019). [in Russian].

3. Tucker R.W., Wang C. Torsional Vibration Control and Cosserat Dynamics of a Drill-Rig Assembly. Meccanica, 2003, Vol. 38, No. 1, pp. 145161. DOI: https://doi.org/10.1023/A:102203582.

4. Christoforou A.P., Yigit A.S. Fully Coupled Vibrations of Actively Controlled Drillstrings. Journal of Sound and Vibrations, 2003, Vol. 267, No. 5, pp. 1029-1045. DOI: 10.1016/S0022-460X(03)00359-6.

5. Sampaio R., Piovan M.T., Venero Lozano G. Coupled Axial/Torsional Vibrations of Drill-Strings by Means of Non-Linear Model. Mechanics Research Communications, 2007, Vol. 34, No. 5-6, pp. 497-502. DOI: 10.1016/j.mechrescom.2007.03.005.

6. Khulief Y.A., Al-Sulaiman F.A., Bashmal S. Vibration Analysis of Drill Strings with Self-Excited Stick-Slip Oscillations. Journal of Sound and Vibration, 2007, Vol. 299, No. 3, pp. 540-558. DOI: 10.1016/j.jsv.2006.06.065. 
7. Yunin E.K. Nizkochastotnye kolebaniya buril'nogo instumenta [LowFrequency Oscillations of Drill Bit]. Moscow, Nedra Publ, 1983. 132 p. [in Russian].

8. Palmov V.A., Vetyukov Yu.A. Neravnomernoe vraschenie byril'noi kolonny obuslovlennoe suschestvenno nelineinym vzaimodeistviem dolota so skal'noi porodoi [Non-Steady Rotation of Drill string Due to Essentially Nonlinear Drill Bit-Rock Interaction]. Nauchno-tekhnicheskie vedomosti SPbGTU - St. Petersburg Polytechnical University Journal, 2003, No. 4, pp. 51-56. [in Rissian].

9. Koronatov V.A. Novaya dinamicheskaya model buril'noi kolonny i chislennoe opisanie krutil'nyh avtokolebanii vo vremya prokhodki - uglubleniya zaboya skvazhiny [New Dynamic Model of a Drill String and Numerical Description of Torsional Self-Oscillations During Penetration - Bottom-Hole Deepening]. Sistemy. Metody. Tekhnologii. - Systems. Methods. Technologies, 2015, No. 1 (25), pp. 48-59. [in Russian].

10. Pogorelov D.Yu., Pavlukov A.E., Yudakova T.A., Kotov S.V. Modelirovanie kontaktnykh vzaimodeistvii $\mathrm{v}$ zadachakh dinamiki system tel [Contact Interaction Modeling in Multibody System Dynamics]. Sbornik nauchnykh trudov «Dinamika, prochnost' i nadezhnost' transportnykh mashin» [Dynamics, Strength and Durability of Transport Machines. Collected Papers] Bryansk, BSTU, 2002. pp. 11-23. [in Russian].

11. Detournay E., Defourny P. A Phenomenological Model for the Drilling Action of Drag Bits. Int. J. Rock Mech. Min., 1992, Vol. 29, No. 1, pp. 13-23.

12. Richard T., Germay C., Detournay E. Self-Excited Stick-Slip Oscillations of Drill Bits. Mecanique, 2004, Vol. 332, pp. 619-626. DOI: https://doi.org/10.1016/j.crme.2004.01.016.

13. Richard T., Germay C., Detournay E. A Simplified Model to Explore the Root Cause of Stick-Slip Vibrations in Drilling Systems with Drag Bits. Journal of Sound and Vibration, 2007, Vol. 305, No. 3, pp. 432-456. DOI: https://doi.org/10.1016/j.jsv.2007.04.015. 
14. Pogorelov D., Mikheev G., Lysikov N., Ring L., Gandikota R., Abedrabbo N. A Multibody System Approach to Drill String Dynamics Modeling. Proc. ASME 11th Biennial Conference on Engineering Systems Design and Analysis (ESDA2012). France, 2012, Vol. 4, pp. 53-62. DOI: 10.1115/ESDA2012-82316.

15. Dmitrochenko O., Mikheev G., Pogorelov D., Gandikota R. A Nonlinear Finite Element for Simulation of Dynamics of Beam Structures Using Multibody System Approach. Proc. 11th World Congress on Computational Mechanics (WCCM XI). Spain, 2014, pp. 655-666.

16. Brakel J.D. Prediction of Wellbore Trajectory Considering Bottom Hole Assembly and Drillbit Dynamics. PhD Thesis, University of Tulsa. 1986.

17. Spanos P.D., Chevallier A.M., Politis N.P. Nonlinear Stochastic DrillString Vibrations. Journal of Vibration and Acoustics, 2002, Vol. 124, No. 4, pp. 512-518. DOI: https://doi.org/10.1115/1.1502669.

18. Grigoryan N.A., Baubhjd R.E. Analiz protsessov turbinnogo bureniya [Analysis of Turbine Motor Drilling Processes]. Moscow, Nedra Publ, 1982. 207 p. [in Russian].

19. Spivak A.I. Popov A.N. Razrushenie gornykh porod pri burenii skvazhin: uchebnik dlya vuzov [Rock Destruction by Borehole Drilling. Tutorial]. Moscow, Nedra Publ., 1994. 264 p. [in Russian].

20. Kalinin A.G., Levitskii A.Z., Messer A.G., Solovyev N.V. Prakticheskoe rukovodstvo po tehnologii bureniya skvazhin na zhidkie i gazoobraznye poleznye iskopaemye [Practical Guidance on Drilling Technology for Oil and Gas Mineral Resources]. Moscow, Nedra Publ., 2001. 450 p. [in Russian].

21. Stribeck R. Die wesentlichen eigenschaften der Gleit- und Rollenlager. VDI-Zeitschrift, 1902, Vol. 46, No. 36, pp. 1341-1348, 1432-1438, 1463-1470.

22. Warren T.M. Penetration-Rate Performance of Roller-Cone Bits. SPE Drilling Engineering, 1987, Vol. 2, No. 1, pp. 9-18. DOI: https://doi.org/10.2118/13259-PA. 
23. Warren T.M. Factors Affecting Torque for a Roller Cone Bit. Journal of Petroleum Technology, 1984, Vol. 36, No. 9, pp. 1500-1508. DOI: https://doi.org/10.2118/11994-PA.

24. Sheppard M.C., Lesage M. The Forces at the Teeth of a Drilling Roller Cone Bit: Theory and Experiment. Society of Petroleum Engineers, 1988, SPE18042, pp. 253-260.

\section{Сведения об авторах}

\section{About the authors}

Погорелов Дмитрий Юрьевич, д-р физ.-мат. наук, профессор, руководитель лаборатории «Вычислительная механика» БГТУ, г. Брянск, Российская Федерация

Dmitriy Yu. Pogorelov, Doctor of Physical and Mathematical Sciences, Professor, Head of Laboratory of Computational Mechanics, Bryansk State Technical University, Bryansk, Russian Federation

e-mail:pogorelov@umlab.ru

Лысиков Николай Николаевич, инженер НИС лаборатории «Вычислительная механика» БГТУ, г. Брянск, Российская Федерация

Nikolay N. Lysikov, Engineer of Laboratory of Computational Mechanics, Bryansk State Technical University, Bryansk, Russian Federation e-mail: lysikov@umlab.ru 